University of Nebraska - Lincoln

DigitalCommons@University of Nebraska - Lincoln

US Department of Energy Publications

U.S. Department of Energy

2010

\title{
Ellipsometric Study of Single-Crystal Y-Inse From 1.5 To 9.2 Ev
}

S. G. Choi

National Renewable Energy Laboratory, Golden, Colorado 80401, USA

D. E. Aspnes

Department of Physics, North Carolina State University, Raleigh, North Carolina 27695, USA

A. L. Fuchser

J.A. Woollam Co. Inc., Lincoln, Nebraska 68508, USA

C. Martinez-Tomas

Departamento de Física Aplicada i Electromagnetismo, Universitat de València, C/Dr. Moliner 50, 46100 Burjassot, Spain

V. Muñoz Sanjosé

Departamento de Física Aplicada i Electromagnetismo, Universitat de València, C/Dr. Moliner 50, 46100

Burjassot, Spain

See next page for additional authors

Follow this and additional works at: https://digitalcommons.unl.edu/usdoepub

Part of the Bioresource and Agricultural Engineering Commons

Choi, S. G.; Aspnes, D. E.; Fuchser, A. L.; Martinez-Tomas, C.; Muñoz Sanjosé, V.; and Levi, D. H., "Ellipsometric Study of Single-Crystal Y-Inse From 1.5 To 9.2 Ev" (2010). US Department of Energy Publications. 65.

https://digitalcommons.unl.edu/usdoepub/65

This Article is brought to you for free and open access by the U.S. Department of Energy at DigitalCommons@University of Nebraska - Lincoln. It has been accepted for inclusion in US Department of Energy Publications by an authorized administrator of DigitalCommons@University of Nebraska - Lincoln. 


\section{Authors}

S. G. Choi, D. E. Aspnes, A. L. Fuchser, C. Martinez-Tomas, V. Muñoz Sanjosé, and D. H. Levi 


\title{
Ellipsometric study of single-crystal $\gamma$-InSe from 1.5 to $9.2 \mathrm{eV}$
}

\author{
S. G. Choi, ${ }^{1, a)}$ D. E. Aspnes, ${ }^{2}$ A. L. Fuchser, ${ }^{3}$ C. Martinez-Tomas, ${ }^{4}$ V. Muñoz Sanjosée, ${ }^{4}$ \\ and D. H. Levi ${ }^{1}$ \\ ${ }^{1}$ National Renewable Energy Laboratory, Golden, Colorado 80401, USA \\ ${ }^{2}$ Department of Physics, North Carolina State University, Raleigh, North Carolina 27695, USA \\ ${ }^{3}$ J.A. Woollam Co. Inc., Lincoln, Nebraska 68508, USA \\ ${ }^{4}$ Departamento de Física Aplicada i Electromagnetismo, Universitat de València, C/Dr. Moliner 50, \\ 46100 Burjassot, Spain
}

(Received 4 January 2010; accepted 9 April 2010; published online 6 May 2010)

\begin{abstract}
We report the component $\vec{E} \perp \hat{c}$ of the pseudodielectric-function tensor $\langle\varepsilon(E)\rangle=\left\langle\varepsilon_{1}(E)\right\rangle+i\left\langle\varepsilon_{2}(E)\right\rangle$ of $\gamma$-phase single-crystal InSe, obtained from 1.5 to $9.2 \mathrm{eV}$ by vacuum-ultraviolet spectroscopic ellipsometry with the sample at room temperature. Overlayer artifacts were reduced as far as possible by measuring fresh surfaces prepared by cleavage. Accurate critical-point energies of observed structures were obtained by a combined method of spectral analysis. () 2010 American Institute of Physics. [doi:10.1063/1.3420080]
\end{abstract}

InSe is a potential material for thin-film solar-cell applications owing to its favorable $(\sim 1.2 \mathrm{eV})$ band gap energy, high $\left(>1000 \mathrm{~cm}^{2} / \mathrm{V} \mathrm{s}\right)$ carrier mobility, long $(>1 \mathrm{~mm}$ within and $5 \mu \mathrm{m}$ across layer) diffusion lengths, and opticalquality cleaved surfaces. ${ }^{1,2}$ With the bulk of the solar spectrum occurring at photon energies greater than $1.2 \mathrm{eV}$, a knowledge of its above-band gap optical properties is obviously important not only to design solar cells but also to verify theoretical calculations of the electronic energy band structure. While a limited number of optical studies have been done on InSe, mainly by reflectance techniques, ${ }^{3-5}$ no systematic investigation of the dielectric response of this material has yet been reported.

Here, we use vacuum-ultraviolet spectroscopic ellipsometry (VUV-SE) to determine the component $\left\langle\varepsilon_{\perp}(E)\right\rangle=\left\langle\varepsilon_{\perp 1}(E)\right\rangle+i\left\langle\varepsilon_{\perp 2}(E)\right\rangle$ of the pseudodielectric function $\langle\varepsilon\rangle$ that is perpendicular to the cleavage plane of $\gamma$-phase single-crystal InSe(001). Data were obtained from 1.5 to $9.2 \mathrm{eV}$ on freshly cleaved surfaces to minimize overlayer effects. The sample was at room temperature. SE is particularly well suited for these measurements because it is highly accurate and allows $\left\langle\varepsilon_{\perp 1}\right\rangle$ and $\left\langle\varepsilon_{\perp 2}\right\rangle$ to be measured simultaneously without need for Kramers-Kronig (K-K) analysis.

$\gamma$-phase InSe crystallizes in a layered structure characterized by strong intralayer binding and relatively weak interlayer binding, as occurs in many other III-VI compounds. The stacking order and energy-band-structure calculations ${ }^{6}$ suggest that there should be in-plane as well as out-of-plane anisotropy, such that InSe is optically biaxial. However, our $\left\langle\varepsilon_{\perp}\right\rangle$ data show no evidence of in-plane anisotropy, consistent with reflectance studies, ${ }^{3-5}$ so $\gamma$-InSe can be regarded to present levels of precision as an optically quasiuniaxial material. $\left\langle\varepsilon_{\perp}\right\rangle$ spectra obtained on an optically uniaxial crystal oriented with the optic axis perpendicular to the crystal surface are a close approximation to the ordinary component $\varepsilon_{\mathrm{o}}$ of the dielectric tensor of the crystal, since the $c$-axis contribution is reduced approximately by $1 /\langle\varepsilon\rangle\rangle^{7,8}$ This approxi-

\footnotetext{
${ }^{\text {a) }}$ Author to whom correspondence should be addressed. Electronic mail: sukgeun.choi@nrel.gov.
}

mation has been shown to be valid for single-crystal $\mathrm{TiO}_{2}$, which is highly anisotropic $(|\Delta n| \approx 0.3)$ below the fundamental absorption edge. ${ }^{8}$ Although a recent SE study ${ }^{9}$ of wurtzite $\mathrm{ZnO}$ and $\mathrm{GaN}$ found a large discrepancy between $\left\langle\varepsilon_{\perp}\right\rangle$ and $\varepsilon_{\mathrm{o}}$ in the transparent and excitonic-absorption regions, the discrepancy above the band gap is relatively small. Therefore, we consider our $\left\langle\varepsilon_{\perp}\right\rangle$ spectra to be a good approximation to $\varepsilon_{0}$. Rigorous accommodation of the $c$-axis component would require the preparation of a specular surface orthogonal to the cleavage plane, which is precluded by the soft nature of the material, as was also found recently for $\mathrm{In}_{4} \mathrm{Se}_{3}{ }^{10}$

We obtain critical-point $(\mathrm{CP})$ energies of the structures observed in our data by a combined reciprocal-/direct-space analysis method, where the data are first filtered in reciprocal space (RS), then transformed back to direct space (DS) as second-energy-derivatives for detailed line shape analysis. ${ }^{11}$ In RS the information is separated from noise, allowing filtering and differentiation to be done without affecting the $\mathrm{CP}$ parameters. ${ }^{12}$ While CP parameters can be obtained entirely in RS, this becomes difficult if the spectral segments being analyzed contain three or more CPs, as is the situation here. Then, it is advantageous to back-transform the filtered data to DS and perform the analysis where the CP structures are better separated. By first separating the information from noise, then separating the critical points themselves, we improve accuracy and avoid systematic errors such as phase reversal and line shape distortions ${ }^{13}$ that occur in leastsquares polynomial methods such as those described by Savitzky and Golay (SG). ${ }^{14}$

The sample was a bulk $\gamma$-phase InSe single crystal grown by the vertical Bridgman method. It was prepared by cutting the ingot into a rectilinear $8 \times 8 \times 0.2 \mathrm{~mm}^{3}$ block with the large faces normal to the [001] direction. The VUV-SE is a rotating-analyzer type equipped with a computer-controlled $\mathrm{MgF}_{2}$ Berek compensator to improve accuracy, particularly below the band gap. The angle of incidence was $67^{\circ}$. Each data point represents an average of 100 analyzer revolutions to enhance the signal-to-noise ratio. Samples were loaded into the SE system as quickly as possible after cleavage. The system was continuously purged 

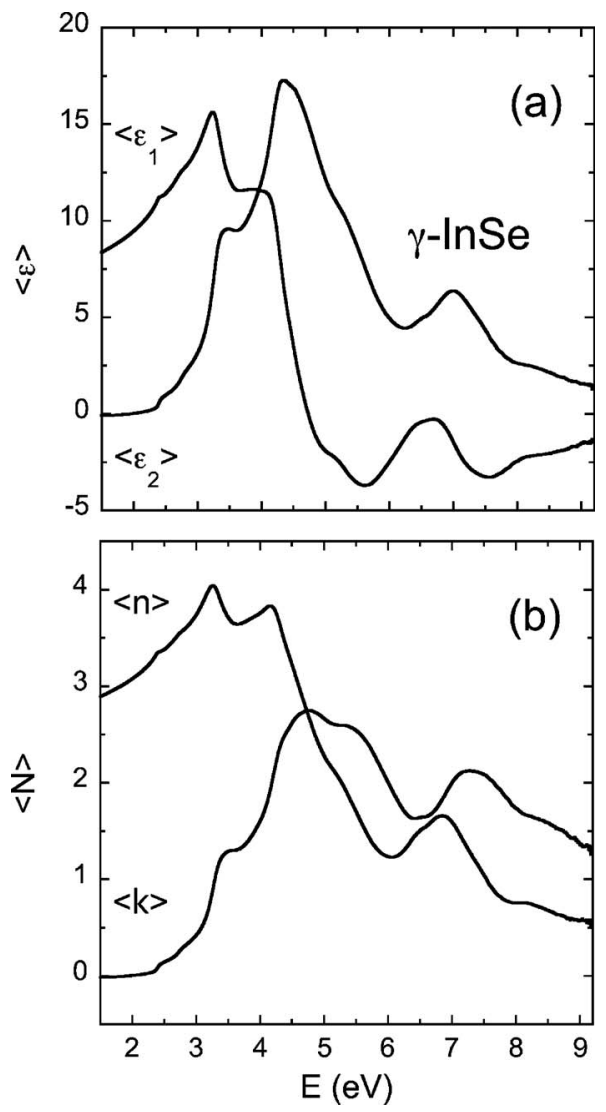

FIG. 1. (a) Pseudodielectric function $\left\langle\varepsilon_{\perp}\right\rangle=\left\langle\varepsilon_{\perp 1}\right\rangle+i\left\langle\varepsilon_{\perp 2}\right\rangle$ and (b) pseudorefractive index $\left\langle N_{\perp}\right\rangle=\left\langle n_{\perp}\right\rangle+\left\langle k_{\perp}\right\rangle$ for single-crystal $\gamma$-phase InSe(001).

with dry $\mathrm{N}_{2}$ not only to protect the sample surface from contamination but also to avoid the absorption of light below $190 \mathrm{~nm}$ by oxygen and water vapor in the ambient.

Figures 1(a) and 1(b) present our $\left\langle\varepsilon_{\perp}\right\rangle=\left\langle\varepsilon_{\perp 1}\right\rangle+i\left\langle\varepsilon_{\perp 2}\right\rangle$ and pseudorefractive index $\left\langle\tilde{N}_{\perp}\right\rangle=\left\langle n_{\perp}\right\rangle+i\left\langle k_{\perp}\right\rangle$ spectra, respectively. The major optical structures near 3.5, 4.5, and $7.0 \mathrm{eV}$ are in good agreement with previously reported $\varepsilon$ spectra obtained by a $\mathrm{K}-\mathrm{K}$ transformation of reflectance data. ${ }^{4,5}$ However, our spectra show several weak structures in the high-energy transition region that were not clearly observed in previous reflectance measurements. ${ }^{4,5}$ This may be due to higher crystalline quality, better minimization of surface artifacts, the advantages in accuracy of SE relative to reflectance, ${ }^{15}$ or a combination of the above.

Figure 2 compares $d^{2}\left\langle\varepsilon_{\perp 1}\right\rangle / d E^{2}$ spectra obtained by different filtering methods to support the above comments on filtering. The light gray line is the reference $d^{2}\left\langle\varepsilon_{\perp 1}\right\rangle / d E^{2}$ spectrum calculated from $\left\langle\varepsilon_{\perp 1}\right\rangle$ by applying the three-pointderivative algorithm twice. This represents the minimum possible smoothing. The blue line is $d^{2}\left\langle\varepsilon_{\perp 1}\right\rangle / d E^{2}$ calculated by SG filtering in DS with the fourth-degree polynomial and 11 data points. The red line is $d^{2}\left\langle\varepsilon_{\perp 1}\right\rangle / d E^{2}$ calculated as described above, with Gaussian filtering in RS. There is no significant difference in line shapes below $7 \mathrm{eV}$, except for the amplitude reduction near $3.25 \mathrm{eV}$ that occurs with DS filtering, as shown more clearly in the inset. However, above $7 \mathrm{eV}$ the signal-to-noise ratio is significantly poorer, and the spectral features are less pronounced. Here, RS filtering offers a genuine benefit. Attempts to reduce the noise level in this spectral region by DS analysis, either by employing

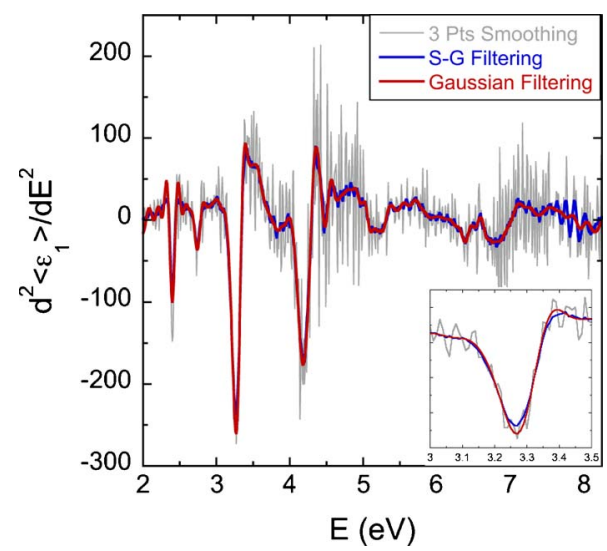

FIG. 2. (Color online) $d^{2}\left\langle\varepsilon_{\perp 1}\right\rangle / d E^{2}$ spectra filtered in different ways. Thin gray line: two applications of three-point differentiation, representing the minimum possible smoothing. Blue line: DS SG filtering with a fourthdegree polynomial and 11 data points. Red line: Reconstructed from coefficients Gaussian-filtered in RS. Inset: same spectra from 3.0 to $3.5 \mathrm{eV}$.

lower-degree polynomials or increasing the filtering range, ${ }^{13}$ unacceptably distort the information and the associated line shapes. These results are not shown.

The CP energies listed in Table I are obtained with the standard analytic CP expressions ${ }^{16}$

$$
\frac{d^{2} \varepsilon}{d E^{2}}=\left\{\begin{array}{c}
n(n-1) A e^{i \phi}\left(E-E_{g}+i \Gamma\right)^{n-2}, n \neq 0 \\
A e^{i \phi}\left(E-E_{g}+i \Gamma\right)^{-2}, n=0
\end{array},\right.
$$

where $A$ is the amplitude, $E_{g}$ is the threshold energy, $\Gamma$ is the broadening parameter, and $\phi$ is the phase. The exponent $n$ has the values $-1,-1 / 2,0$, and $1 / 2$ for excitonic, one-, two-, and three-dimensional line shapes, respectively. Both real and imaginary parts were fit simultaneously. The open circles in Fig. 3 give the $d^{2}\left\langle\varepsilon_{\perp 1}\right\rangle / d E^{2}$ spectrum reconstructed from Gaussian-filtered RS coefficients as described above. For clarity we show only one-third of the $d^{2}\left\langle\varepsilon_{\perp 1}\right\rangle / d E^{2}$ circles and none of those for $d^{2}\left\langle\varepsilon_{\perp 2}\right\rangle / d E^{2}$. The solid and dasheddotted lines represent the best-fit curves of the real and imaginary parts, respectively, for the excitonic line shape $n$ $=-1$. This value of $n$ gave the best fit, and was used to obtain the CP energies listed in Table I. Although not shown, the quality of the fit to $d^{2}\left\langle\varepsilon_{\perp 2}\right\rangle / d E^{2}$ is similar.

TABLE I. CP energies of InSe, in eV. Values are for room temperature except those denoted with "b."

\begin{tabular}{lcccc}
\hline \hline \multicolumn{1}{c}{ CP } & This work & Ref. 20 & Ref. 3 & Ref. 4 \\
\hline$E_{A}$ & 2.40 & 2.30 & 2.44 & $2.33^{\mathrm{b}}$ \\
$E_{B}$ & 2.75 & 2.70 & 2.83 & $2.90^{\mathrm{b}}$ \\
$E_{C}$ & 3.31 & 3.40 & 3.35 & 3.45 \\
$E_{D}$ & 3.48 & & & \\
$E_{E}$ & 4.07 & 3.90 & 3.91 & \\
$E_{F}$ & 4.23 & & & \\
$E_{G}$ & 4.49 & 4.40 & 4.40 & 4.45 \\
$E_{H}$ & 4.96 & & 4.70 & 4.70 \\
$E_{I}$ & 5.13 & 5.20 & & 5.00 \\
$E_{J}$ & 6.51 & 6.30 & 6.30 & 5.70 \\
$E_{K}$ & 6.96 & 6.60 & & 7.00 \\
$E_{L}$ & 7.41 & 7.40 & 7.60 & \\
\hline \hline
\end{tabular}

${ }^{\mathrm{a}}$ Theoretical study.

${ }^{\mathrm{b}}$ Measured at liquid He temperature. 


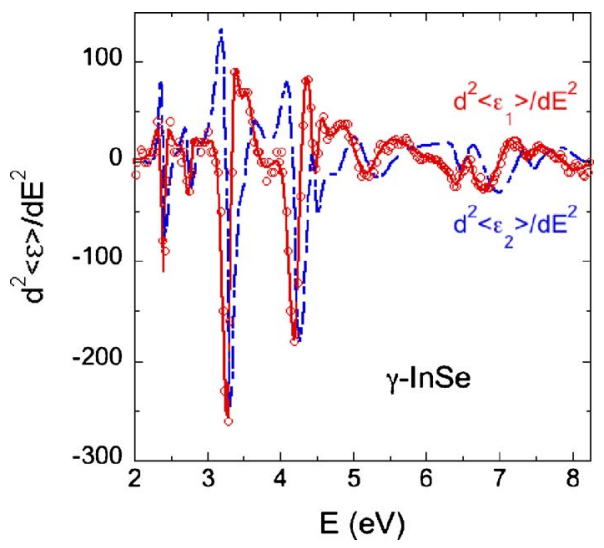

FIG. 3. (Color online) Red and blue solid lines: best-fit curves for $d^{2}\left\langle\varepsilon_{\perp 1}\right\rangle / d E^{2}$ and $d^{2}\left\langle\varepsilon_{\perp 2}\right\rangle / d E^{2}$, respectively, of InSe. Open circles: real part of the corresponding data filtered in RS. For clarity only one-third of the circles are shown.

The complicated nature of the energy band structure of InSe and related materials makes the unambiguous identification of the origins of the structures observed experimentally a difficult challenge. ${ }^{3}$ Nevertheless, there is a consensus that the first two above-band gap CP structures, $E_{A}$ and $E_{B}$ at 2.40 and $2.75 \mathrm{eV}$ in Table I, result from the excitation of Se $p_{x} p_{y}$ electrons to the conduction band minimum at the $\Gamma$ point of the Brillouin zone (BZ)., ${ }^{47,18}$ The energy separation results from the spin-orbit splitting of the $\mathrm{Se} p_{x} p_{y}$ bands, which from our data is $0.35 \mathrm{eV}$. Our data also show that the energy separation between the top valence band, of Se $p_{z}$ character, and the next highest valence band, of Se $p_{x} p_{y}$ character, is $1.2 \mathrm{eV}$ at the $\Gamma$ point. We obtain this by subtracting the $1.2 \mathrm{eV}$ energy ${ }^{19}$ of the fundamental absorption edge from the $2.4 \mathrm{eV}$ energy that we observe for $E_{A}$. This value is in excellent agreement with a previous opticalabsorption study ${ }^{18}$ and theoretical predictions. ${ }^{18}$

The BZ origins of the higher-energy CPs remain uncertain. Piacentini et $a l^{4}{ }^{4}$ attributed the $E_{C} \mathrm{CP}$ at $3.31 \mathrm{eV}$ to transitions from the valence band maximum to the third group of conduction bands at or near the $\Gamma$ point but also considered other possibilities. They assigned the broad band of $\mathrm{CP}$ structures from 4 to $6 \mathrm{eV}$ to excitation of Se $p_{x} p_{y}$ electrons to either the second or the third group of conduc- tion bands. In contrast, Mamy and Couget ${ }^{5}$ suggested that these structures are due to CPs involving deeper valence bands consisting of In $5 s$ and Se $4 s$ states. Further systematic theoretical investigations are necessary to resolve these issues. One of our goals is to provide theoreticians with information needed to perform these calculations for InSe and other III-VI compounds.

This work was supported by the U.S. Department of Energy under Contract No. DE-AC36-08GO28308. The work done at the Universitat de València was supported in part by the Spanish Project under Grant No. MAT2007-66129.

${ }^{1}$ A. Segura, J. P. Guesdon, J. M. Besson, and A. Chevy, J. Appl. Phys. 54, 876 (1983).

${ }^{2}$ J. F. Sánchez-Royo, A. Segura, O. Lang, E. Schaar, G. Pettenkofer, W. Jaegermann, L. Roa, and A. Chevy, J. Appl. Phys. 90, 2818 (2001).

${ }^{3}$ V. V. Sobolev and V. I. Donetskich, Phys. Status Solidi B 45, K15 (1971).

${ }^{4}$ M. Piacentini, C. G. Olson, A. Balzarotti, R. Girlanda, V. Grasso, and E. Doni, Nuovo Cimento 54B, 248 (1979).

${ }^{5}$ R. Mamy and A. Couget, Solid State Commun. 32, 1129 (1979).

${ }^{6}$ P. Gomes da Costa, R. G. Dandrea, R. F. Wallis, and M. Balkanski, Phys. Rev. B 48, 14135 (1993).

${ }^{7}$ D. E. Aspnes, J. Opt. Soc. Am. 70, 1275 (1980).

${ }^{8}$ J. E. Jellison, Jr. and J. S. Baba, J. Opt. Soc. Am. A Opt. Image Sci. Vis 23, 468 (2006).

${ }^{9}$ S. Shokhovets, L. Spieß, and G. Gobsch, J. Appl. Phys. 107, 023509 (2010).

${ }^{10}$ L. Makinistian, E. A. Albanesi, N. V. Gonzalez Lemus, A. G. Petukhov, D. Schmidt, E. Schubert, M. Schubert, Ya. B. Losovyj, P. Galiy, and P. Dowben, Phys. Rev. B 81, 075217 (2010).

${ }^{11}$ S. G. Choi, D. E. Aspnes, N. A. Stoute, Y. D. Kim, H. J. Kim, Y.-C Chang, and C. J. Palmstrøm, Phys. Status Solidi A 205, 884 (2008).

${ }^{12}$ S. D. Yoo and D. E. Aspnes, J. Appl. Phys. 89, 8183 (2001).

${ }^{13}$ P. Marchand and L. Marmet, Rev. Sci. Instrum. 54, 1034 (1983).

${ }^{14}$ A. Savitzky and M. J. E. Golay, Anal. Chem. 36, 1627 (1964).

${ }^{15}$ D. E. Aspnes, in Handbook of Optical Constants of Solids, edited by E. D. Palik (Academic, Orlando, 1985).

${ }^{16}$ M. Cardona, Modulation Spectroscopy, Solid State Physics Suppl. 11, edited by F. Seitz, D. Turnbell, and H. Ehrenreich (Academic, New York, 1969).

${ }^{17}$ V. Grasso and G. Mondio, in Electronic Structure and Electronic Transitions in Layered Materials, edited by V. Grasso (D. Reidel, Dordrecht, 1986).

${ }^{18}$ N. Kuroda and Y. Nishina, Physica B \& C 105, 30 (1981).

${ }^{19}$ M. V. Andriyashik, M. Yu. Saknovskii, V. B. Timofeev, and A. S. Yakimova, Phys. Status Solidi 28, 277 (1968).

${ }^{20}$ J. V. McCanny and R. B. Murray, J. Phys. C 10, 1211 (1977). 